\title{
Thermal Transport in Molecular Forests
}

\author{
Aashish Bhardwaj, ${ }^{1}$ A. Srikantha Phani, ${ }^{1}$ Alireza Nojeh, ${ }^{2,3}$ and Debashish Mukherji ${ }^{3, *}$ \\ ${ }^{1}$ Department of Mechanical Engineering, University of British Columbia, Vancouver BC V6T 1Z4, Canada \\ ${ }^{2}$ Department of Electrical and Computer Engineering, \\ University of British Columbia, Vancouver BC V6T 1Z4, Canada \\ ${ }^{3}$ Quantum Matter Institute, University of British Columbia, Vancouver BC V6T 1Z4, Canada
}

In this supplementary material we provide supporting data for the results presented in the main manuscript text.

\section{S1. SYSTEM DETAILS}

The polymer brush configurations are generated by randomly tethering the first monomer of each chain onto a plane with lateral dimensions $L_{x}=L_{y} \simeq 36.5 d$ and then chains are created normal to the plane, i.e., along the $z$ direction. All surface coverage concentrations $\Gamma$ are much larger than the critical surface coverage $\Gamma^{*}$ of a polymer chain of $N_{\ell}=500$ onto a plane. Here, $\Gamma^{*}=\left(d / 2 R_{\mathrm{g}}\right)^{2}$, i.e., one chain grafted within an area of $\pi R_{\mathrm{g}}^{2}$. For an isolated chain of $N_{\ell}=500$, the gyration radius $R_{\mathrm{g}} \simeq 18.0 d$. The brush height $\mathcal{H}$ is calculated by taking the mid point of decaying monomer density profile $\rho$ shown in Fig. S1.

TABLE S1: A table listing the system size details used for the simulations. For this purpose a chain length of $N_{\ell}=500$ is chosen. We give details of the grafting density $\Gamma$, the critical grafting density $\Gamma^{*}$, the number of grafted chains $N_{\mathrm{g}}$, the total number of particles $N_{\ell} N_{\mathrm{g}}$, and the brush height $\mathcal{H}$.

\begin{tabular}{|c|c|c|c|c|}
\hline$\Gamma$ & $\Gamma / \Gamma^{*}$ & $N_{\mathrm{g}}$ & $N_{\ell} N_{\mathrm{g}}$ & $\mathcal{H}[d]$ \\
\hline \hline 0.06 & 77.8 & 100 & 50000 & 355 \\
0.13 & 168.5 & 225 & 112500 & 360 \\
0.21 & 272.3 & 361 & 180500 & 380 \\
0.29 & 375.8 & 484 & 242000 & 420 \\
0.65 & 842.4 & 1089 & 544500 & 460 \\
\hline
\end{tabular}

While we have discussed the system equilibration protocol in the main manuscript draft, here we also want to discuss the non-trivial trend observed in Fig. S1. For example, it can be seen that $\rho$ profiles show humps at the top interfaces. On the contrary, a previous prediction had suggested a parabolic decay of $\rho$ profile [1], which is qualitatively different than the data in Fig. S1. Furthermore, the parameter regime where the parabolic profile is observed, the osmotic pressure follows the quadratic dependence with the chain concentration above the chain overlap. However, for the highly compressed brushes, the behavior becomes non-universal and the parabolic decay behavior does not hold [2]. All the brushes used in this

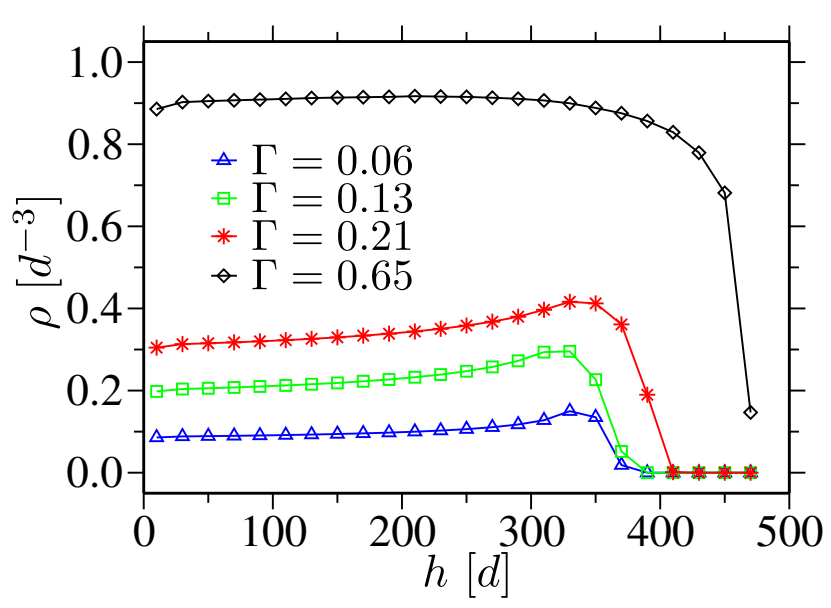

FIG. S1: Monomer number density $\rho$ as a function of height $h$ along the $z$-direction. Data are shown for three different surface coverages $\Gamma$.

study fall under the highly compressed regime, see Table S1. A closer look at the $\rho$ profiles, it becomes clear that the majority contribution to the humps at the free (top) interfaces is populated by the chain ends, see the simulation snapshots in Fig. 2 of the main manuscript text. The segregation of the chain ends at the interfaces for the highly dense systems is not surprising given that the systems always tend to minimize the conformational entropy loss $[3-5]$.

\section{S2. THERMAL CONDUCTIVITY CALCULATIONS}

In Fig S3 we show $h$ dependent $\kappa_{\|}$and $\kappa_{\perp}$. Two important observations can be seen from this data:

(1) While $\kappa_{\|}$decreases with $h, \kappa_{\perp}$ shows an increasing trend with $h$. This is consistent with the second Legendre polynomial $P_{2}$ data shown in Fig. 2(a) of the main manuscript text. More specifically, given the fact that bonds become more-and-more random with $h$, this on one hand reduces the dominant bonded contribution to $\kappa_{\|}$and on the other hand starts contributing more to $\kappa_{\perp}$. Furthermore, both $\kappa_{\|}$and $\kappa_{\perp}$ remain almost invariant for $\Gamma=0.65$, which is not surprising given that $\rho$ and $P_{2}$ remains constant for $h \leq 400 d$, see Fig. S1 and Fig. 2 (a) in the main manuscript text.

(2) For $h \leq 200 d, \kappa_{\|}(\Gamma=0.65) / \kappa_{\|}(\Gamma=0.13) \simeq 2$ 


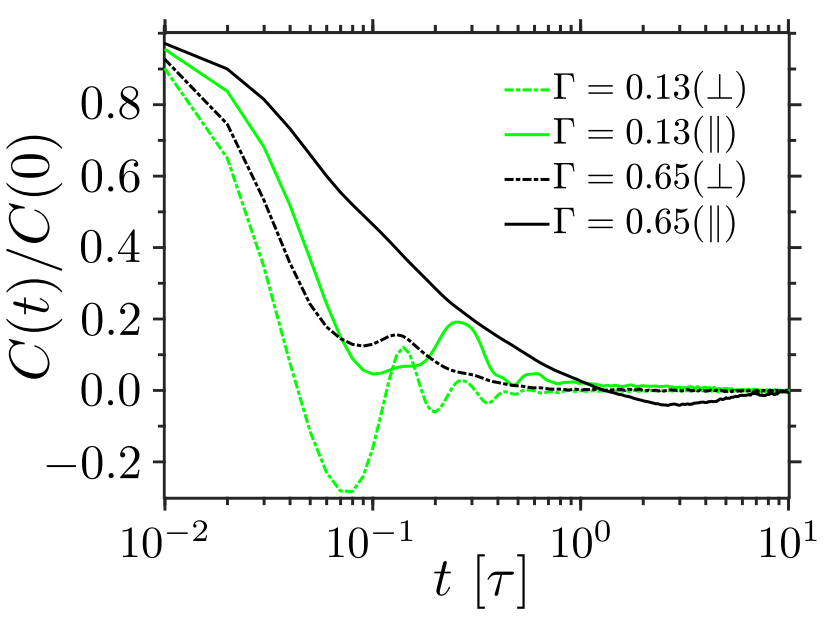

FIG. S2: Normalized heat flux autocorrelation function $C(t) / C(0)$. Data and shown for two different surface coverages $\Gamma$ and for both components. Here we have considered a slab centered on $125 d$.

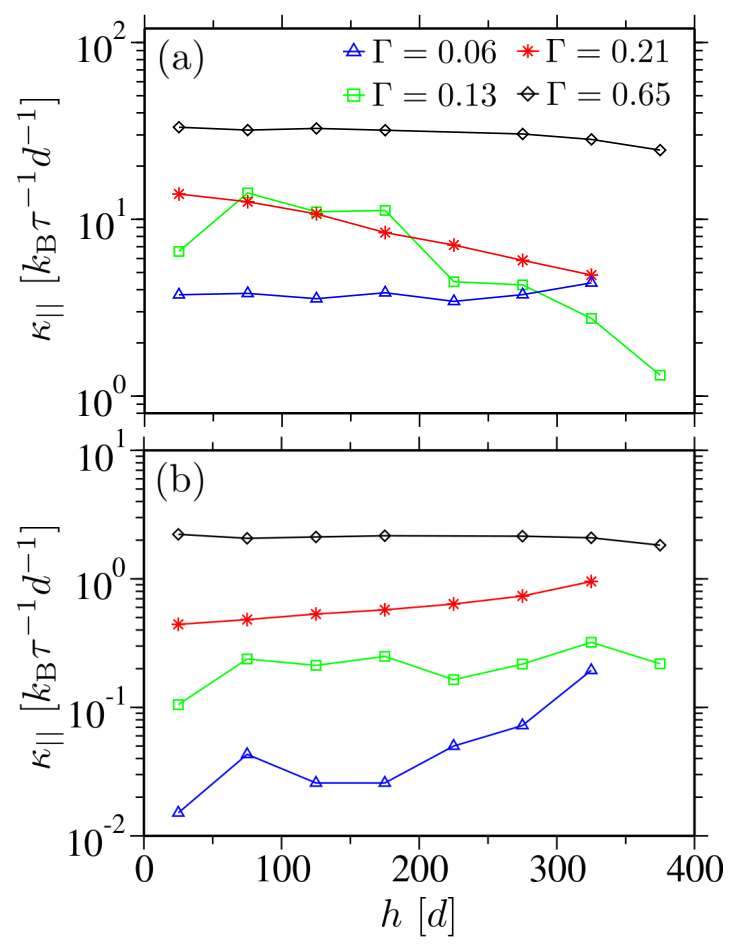

FIG. S3: The parallel $\kappa_{\|}$(a) and perpendicular $\kappa_{\perp}$ (b) components of the thermal conductivity coefficients as a function of height $h$ along the $z$ direction. Data are shown for three different surface coverage concentrations $\Gamma$.

and $\kappa_{\perp}(\Gamma=0.65) / \kappa_{\perp}(\Gamma=0.13) \simeq 10$. These indicate that the increase in $\kappa_{\|}$with $\rho$ is slower than for $\kappa_{\perp}$. This behavior also controls why the $\kappa_{\| \mid} / \kappa_{\perp}$ becomes less anisotropic with $\rho$, see Fig. 3 in the main manuscript text.

\section{S3. TEMPERATURE DEPENDENT THERMAL CONDUCTIVITY}

In the main manuscript text we have highlighted that the $\kappa$ behavior is amorphous like. In Fig. S4 we show that $\kappa$ increases with temperature, which is indeed characteristic of amorphous systems.

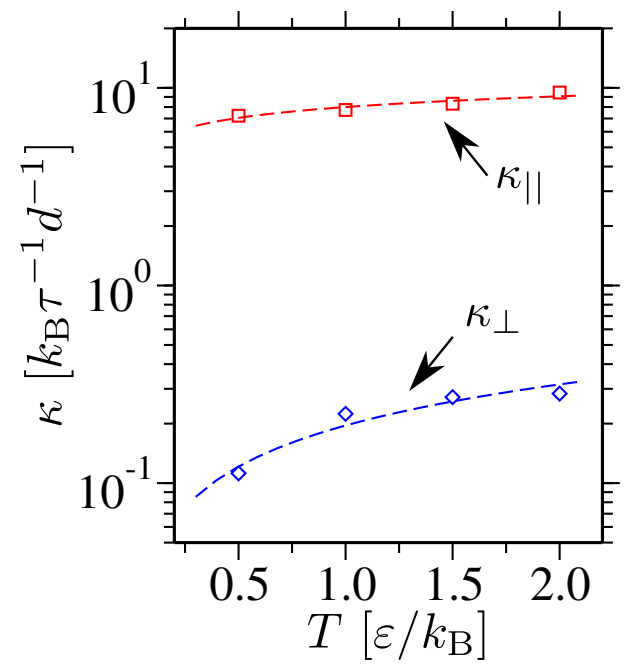

FIG. S4: The parallel $\kappa_{\|}$and perpendicular $\kappa_{\perp}$ components of the thermal conductivity coefficients as a function of temperature $T$. The data are shown for the surface coverage concentration $\Gamma=0.13$. The lines are power law fits to the data that are drawn to guide the eye.

debashish.mukherji@ubc.ca

[1] Milner, S. T.; Witten, T. A.; Cates, M. E. A Parabolic Density Profile for Grafted Polymers. Euro Phys. Lett. 1988, 5, 413 .

[2] Manav, M.; Ponga, M.; Phani, A. S. Stress in a polymer brush. J. Mech. Phys. Sol. 2019, 127, 125.

[3] Bitsanis, I.; Hadziioannou, G. Molecular dynamics simulations of the structure and dynamics of confined polymer melts. J Chem. Phys. 1990, 92, 3827.

[4] Zhao, W.; Zhao, X.; Rafailovich, M. H.; Sokolov, J.; Composto, R. J.; Smith, S. D.; Russell, T. P.; Dozier, W. D.; Mansfield, T.; Satkowski, M. Segregation of chain ends to polymer melt surfaces and interfaces. Macromolecules 1993, 26, 561 .

[5] Wu, D.; Fredrickson, G.; Carton, J.-P.; Ajdari, A.; Leibler, L. Distribution of Chain Ends at the Surface of a Polymer Melt: Compensation Effects and Surface Tension. J Pol. Sci.: Part B: Pol. Phys. 1995, 33, 2373. 